\title{
Une forme de $\beta$-thalassémie avec inclusions cellulaires, à transmission dominante
}

On sait actuellement que les $\beta$ thalassémies, qui sont des maladies génétiques extrêmement répandues, peuvent être dues à un très large spectre de mutations différentes. Certains caractères sont cependant communs à presque toutes les formes. Ce sont, dans l'ensemble, des maladies cliniquement récessives. L'existence de deux gènes $\beta$-globine déficients est nécessaire pour qu'il y ait une traduction pathologique ; les sujets hétérozygotes, en revanche, sont asymptomatiques, et l'on observe chez eux qu'un ensemble discret de signes biologiques. Le déséquilibre de synthèse des chaînes $\alpha /$ non- $\alpha$ de globine est sans doute réduit au cours de la maturation érythrocytaire par une protéolyse des chaînes $\alpha$ en excès, empêchant ainsi une précipitation de monomères $\alpha$ et une destruction cellulaire. Tout au plus, quelques cas ont-ils été signalés où une triplication des gènes $\alpha$ sur un (ou deux) chromosome(s) accentuait le déséquilibre et - de ce fait - majorait la symptomatologie.

Depuis 1973, cependant, des cas isolés ont été décrits qui ne répondent pas à ce tableau classique. Avec des variantes, le tableau clinique observé comporte des signes communs aux thalassémies intermédiaires : anémie le plus souvent modérée autour de 8 ou $10 \mathrm{~g} / \mathrm{dl}$, microcytose, augmentation des hémoglobines $\mathrm{F}$ et $\mathrm{A}_{2}$, déséquilibre de synthèse des chaînes de globine. Plus caractéristique est la splénomégalie importante, pouvant entraîner des besoins transfusionnels par hypersplénisme, et l'hyperplasie érythroïde majeure, qui s'accompagne d'une hématopoièse extramédullaire, d'une hémosidérose à prédominance parenchymateuse et de la présence d'inclusions intracellulaires dès le stade des précurseurs.

Surtout, tous ces signes sont à trans- seul des parents.

Un travail récent, portant sur quatre familles d'Europe du Nord sans relation entre elles, a pu préciser et interpréter les bases moléculaires de ce syndrome clinique et hématologique [1]. Dans trois cas, une mutation GAA $\rightarrow$ TAA au codon $121 \mathrm{du}$ gène de $\beta$-globine crée un codon stop et entraîne l'arrêt de la traduction. Le quatrième cas est dû à une mutation plus complexe : remaniement profond débutant au codon 128, comportant deux délétions de quatre et onze paires de bases, ainsi que l'inclusion de cinq nouvelles bases, avant que la séquence normale ne soit rétablie. Ceci se traduit par un segment de séquence anormale et, ultérieurement, un décalage de phase de lecture ( - $10 \mathrm{pb}$ ) qui passe outre au codon stop normal en position 147, jusqu'au niveau d'un codon stop 153. La chaîne protéique ainsi synthétisée, du codon 128 au codon 153, est exceptionnellement hydrophobe et comporte quatre prolines, dont une en position 128. Elle est de ce fait, à partir de cette proline, incapable de prendre la structure tridimensionnelle normale. A l'appui de cette hypothèse d'une hyperinstabilité de la chaîne protéique formée, on a constaté que, si le rapport de synthèse des chaînes $\alpha /$ non- $\alpha$ est déséquilibré, le rapport des ARN messagers $\alpha /$ non- $\alpha$ est normal, égal à 1,4 .

L'interprétation de ces résultats s'est faite à partir de la connaissance de la structure tridimensionnelle de l'hémoglobine, appuyée par les données de la littérature. La mutation $\mathrm{G} \rightarrow \mathrm{T}$ au codon 121 a en effet été retrouvée dans d'autres cas isolés, toujours accompagnée du même syndrome. Par ailleurs, d'autres cas de syndrome thalassémique avec dysérythropoièse majeure ont été décrits, dans lesquels on a pu iden- tifier une hémoglobine hyperinstable dont la mutation se situait dans la même région du troisième exon de la chaîne $\beta$. D'autres mutations, en revanche, plus proches de l'extrémité C-terminale, ne s'accompagnent que d'une symptomatologie minime. Enfin, les nombreux cas où l'on a pu identifier un codon non-sens, ou une addition/délétion entraînant un décalage de phase de lecture et un arrêt de la traduction en amont du codon 72 , se traduisent par une $\beta$ thalassémie tout à fait classique à transmission récessive, dont la forme hétérozygote est pratiquement asymptomatique.

L'explication moléculaire semble très cohérente. Toute mutation entraînant une terminaison de la traduction avant le codon 72 donne naissance à une chaîne de $\beta$-globine tronquée en amont des sites de fixation de l'hème. Cette chaîne ne forme sans doute pas de structure secondaire et est détruite immédiatement. Le phénotype qui en résulte est celui d'une thalassémie hétérozygote: anémie discrète, dysérythropoièse minimale. $\mathrm{Si}$, au contraire, le codon non-sens est situé au niveau du troisième exon, la traduction de l'ARN messager donne naissance à une chaîne tronquée, ou anormale plus longue, qui s'associe à une molécule d'hème. Une mutation presque terminale, comme il en a été décrit plusieurs donnant lieu à des chaînes allongées, permet la formation d'un tétramère $\alpha_{2} \beta_{2}$ viable. C'est le cas des hémoglobines Tak ou Cranston. Là aussi, les conséquences phénotypiques seront réduites. Mais un certain nombre de mutations s'échelonnent au long du troisième exon. Le cas le plus typique est celui de la mutation non-sens du codon 121, qui entraîne l'arrêt de la traduction avant les résidus destinés à former l'hélice $\mathrm{H}$ de la chaîne $\beta$. Dans la chaîne $\beta$ ainsi

$$
\mathrm{m} / \mathrm{s} n^{\circ} 4 \text {, vol. 7, auril } 91
$$




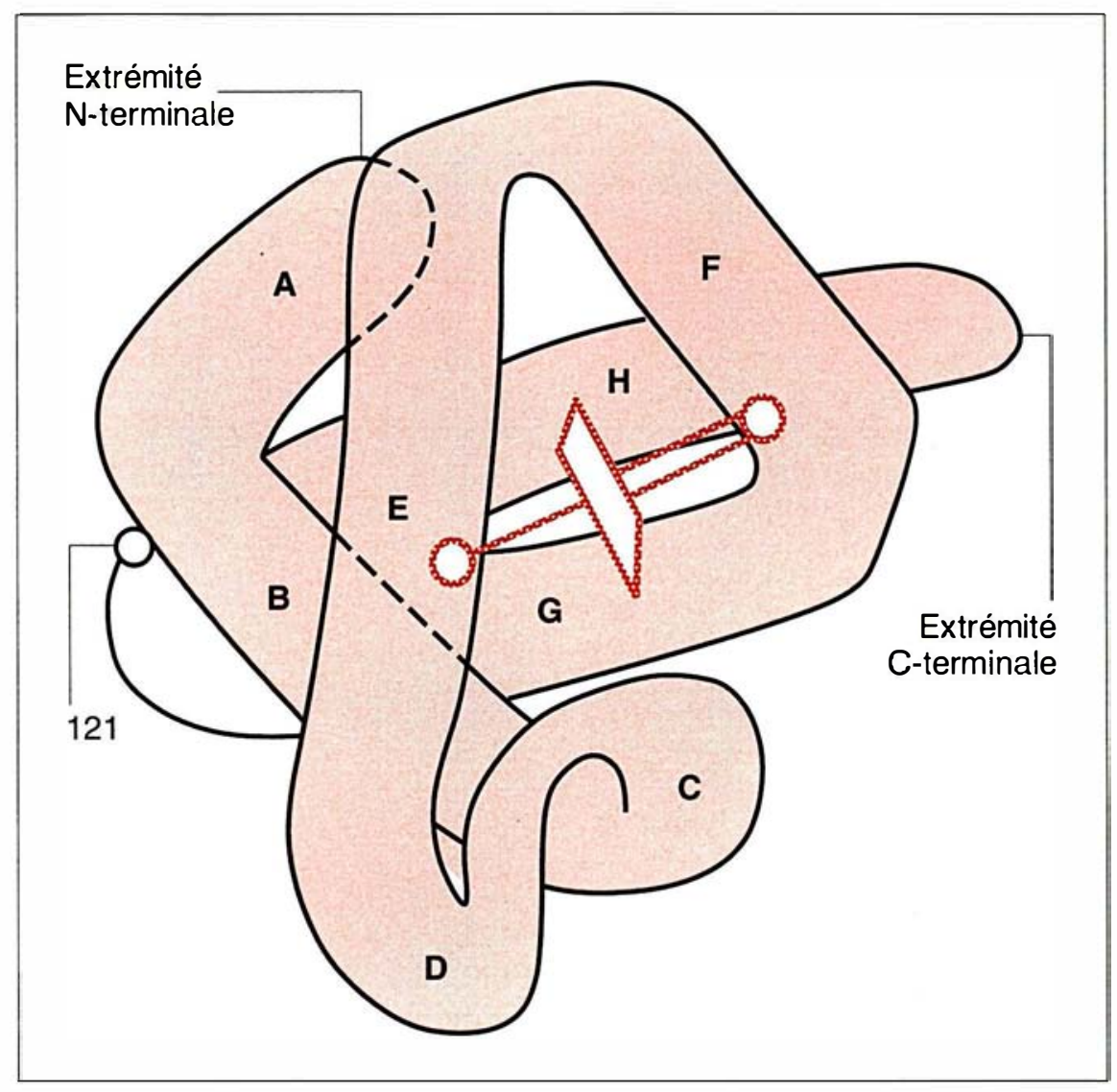

Figure 1. Représentation schématique d'une chaîne de $\beta$-globine en vue externe. La chaîne linéaire est enroulée en une série d'hélices, désignées de $A$ à $H$, qui se replient sur elles-mêmes pour former une structure globulaire. L'hème est enfoui dans une cavité, entre les hélices $E, F$ et $G$ (I'histidine proximale est sur I'hélice F, I'histidine distale sur I'hélice E). L'hélice $H$ interne protège la face postérieure de l'hème contre un contact avec le milieu ambiant aqueux. Le résidu 121 est situé tout au début de cette hélice $H$.

tronquée, les portions hydrophobes des hélices $G, E$ et $F$ se trouvent anormalement exposées, ce qui doit entraîner leur précipitation, sans qu'un tétramère ait pu se former. Il en est vraisemblablement de même quand un décalage de phase de lecture se traduit par une chaîne très hydrophobe et riche en prolines, incapable par conséquent de prendre la conformation d'une hélice $\mathrm{H}$ normale. La revue de plusieurs cas de la littérature, dont les mutations se situent toutes dans la même région, montre qu'il y a prédominance du phénotype thalassémique ou présence d'une hémoglobine anormale plus ou $m / s n^{\circ}$ f, vol. 7, avril 91
1. Thein SL, Hesketh C, Taylor P, et al. Molecular basis for dominantly inherited inclusion body $\beta$-thalassemia. Proc Natl Acad Sci USA $1990 ; 87: 3924-8$. moins instable, suivant la nature de Ce sont sans doute les seules thalassémies présentant un phénotype encontre qu'à l'état sporadique, une transmission dominante.

\section{口 BRÈVES $\square \square$}

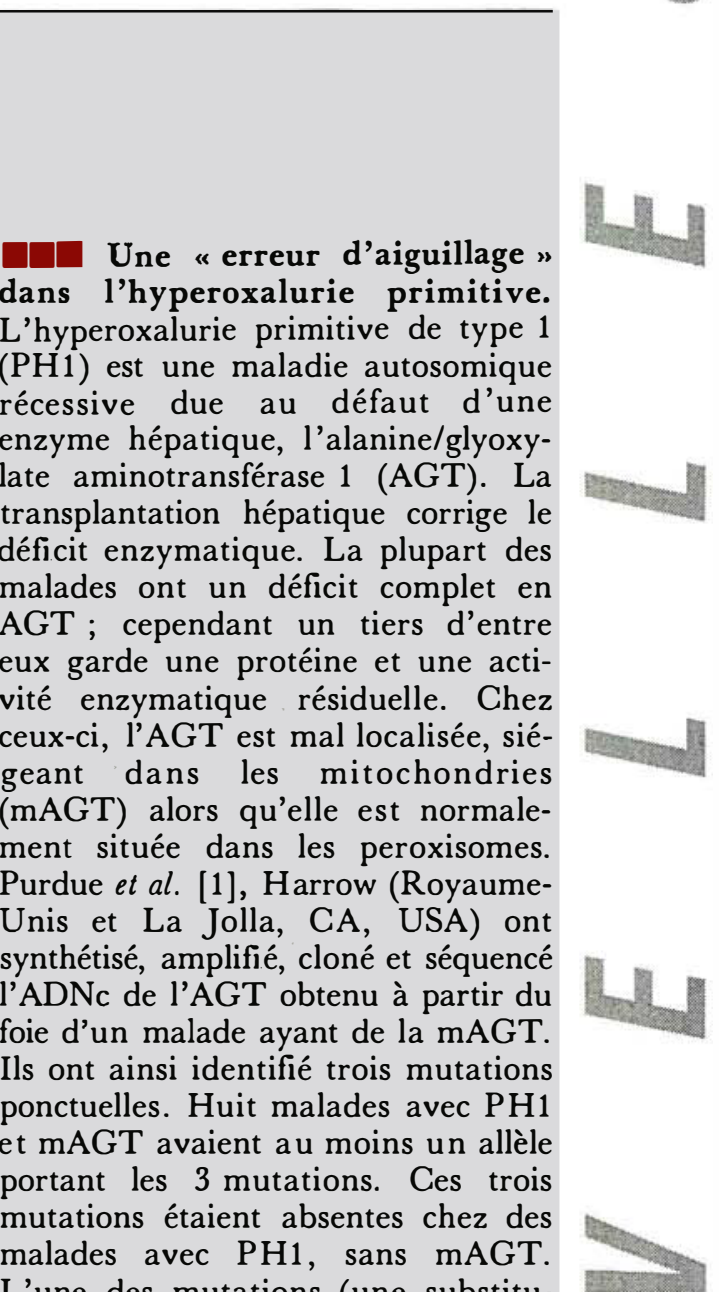
L'une des mutations (une substitution Gly $\rightarrow$ Arg en position 170) n'a jamais été détectée chez les témoins. En revanche les deux autres mutations (Pro $\rightarrow$ Leu et Ile $\rightarrow$ Met, respectivement en positions 11 et 340 ) subissent une co-ségrégation dans la population normale, à une fréquence allélique de 5 à $10 \%$. Chez un sujet homozygote pour cet allèle (substitutions en position 11 et 340 ), seule une faible proportion $(<10 \%)$ de l'AGT est "déroutée " vers les mitochondries. Les auteurs suggèrent que la substitution en position 11 engendre la structure nécessaire à la localisation mitochondriale de l'enzyme, mais que l'expression complète de cette anomalie dépend également de

D. L. la co-expression de la substitution en position 170 qui déterminerait le défaut de transport et de captation dans les peroxisomes.

[1. Purdue PE, et al. J Cell Biol

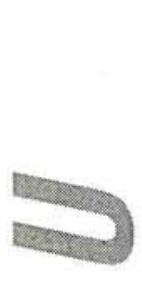

\section{0 ; 111 : 2341-51.]}




\section{aII BRÈVES MTI}

प L'expression d'une kératine tronquée chez des souris transgéniques provoque une désorganisation profonde de l'épiderme évoquant des épidermolyses bulleuses simplex. Chez les vertébrés, la famille des kératines représente le plus grand groupe de protéines des filaments intermédiaires. Ces filaments sont formés par polymérisation d'hétérodimères constitués d'un représentant des kératines de type I, plus petites et plus acides, et d'un représentant des kératines de type II, plus basiques et plus grandes. Dans les cellules basales des épithéliums stratifiés squameux, les filaments intermédiaires sont ainsi constitués de kératine de type I, K14, et de kératine de type II, K5. C'est aussi le cas dans les cellules basales de l'embryon. Lors de la différenciation des kératinocytes et de leur migration vers les couches suprabasales, d'autres couples de kératines sont spécifiquement exprimés. Leur participation au maintien de l'architecture cellulaire et leur production massive dans des cellules qui assurent un rôle essentiel de protection suggèrent qu'elles jouent un rôle important dans le maintien de l'intégrité structurale, et donc fonctionnelle, de ces cellules. L'absence de maladie héréditaire due à une altération de ces molécules n'a cependant pas permis de préciser leur fonction exacte. C'est en se fondant sur l'apparition d'altérations dominantes du cytosquelette provoquée par l'introduction, dans des cellules en culture, d'un gène humain codant pour une kératine $\mathrm{K} 14$ tronquée, que $\mathrm{R}$. Vassar et al., de l'université de Chicago (IL, USA) [1], ont créé des souris transgéniques exprimant ce gène tronqué dans les cellules basales de l'épiderme, lieu d'expression du gène normal. Les souris obtenues ont d'importantes anomalies de l'épiderme et meurent prématurément. Des agrégats de kératine sont observés dans les cellules basales de l'épiderme, siège d'une importante cytolyse provoquant l'apparition de lules différenciées du stratum corneum, qui n'expriment normalement pas le gène $K 14$, la formation des filaments intermédiaires n'est pas altérée, suggérant que ces cellules se différencient précocement à partir de cellules basales qui n'ont pas encore exprimé fortement le transgène. Les études anatomopathologiques réalisées sur les animaux transgéniques exprimant la kératine K14 tronquée montrent la très grande analogie des anomalies observées avec celles des formes les plus sévères d'un groupe hétérogène de maladies de la peau appelées épidermolyses bulleuses simplex (EBS), notamment des formes herpétiques des EBS. Il est à remarquer que dans une étude ultrastructurale réalisée en 1961 [2], la désorganisation des filaments de kératine, dans quelques cas de EBS, était déjà rapportée par les auteurs. L'ensemble de ces résultats justifie pleinement une recherche de mutations des gènes kératines spécifiques des cellules basales, dans les épidermolyses bulleuses graves. Il s'agirait de néomutations dominantes létales expliquant l'absence de formes familiales. Plus généralement, d'autres types d'épidermolyses affectant des cellules suprabasales pourraient être liées à des mutations des gènes kératines spécifiquement exprimés dans des kératinocytes différenciés. Ce pourrait être le cas de certaines épidermolyses hyperkératosiques dans lesquelles les cellules basales sont normales mais la couche suprabasale atteinte. [1. Vassar R, et al. Cell 1991; 64 : 365-80.]

[2. Pearson RW, et al. J Invest Dermatol $1961 ; 36$ : 213-24.]

Les souris transgéniques porteuses de la translocation $\mathrm{t}(14$; 18) constituent d'excellents modèles animaux de la progression tumorale des lymphomes humains liés à cette translocation. La translocation $\mathrm{t}(14 ; 18)$ est retrouvée dans $85 \%$ des lymphomes folliculaires B et dans $20 \%$ des lymphomes diffus.
Elle se produirait précocement au cours du développement des cellules pré-B et aurait pour résultat la juxtaposition du locus des chaînes lourdes des immunoglobulines et du gène $b c l-2$ dont l'expression se trouve perturbée. Le gène $b c l-2$ code pour une protéine de la membrane interne de la mitochondrie qui bloque le mécanisme de mort programmée des lymphocytes appelé apoptose $(\mathrm{m} / \mathrm{s}$ $n^{\circ}$ 1, vol. 7, p. 88) [1]. T. McDonnell et al. ont produit en 1989 des souris transgéniques pour cette translocation. Le transgène bcl-2-Ig est exprimé dans les tissus lymphoïdes des animaux transgéniques et provoque un allongement de la durée de vie des cellules $B$ matures à l'origine d'une hyperplasie lymphoïde atypique, polyclonale, détectable entre 8 et 25 semaines [2]. Plus tardivement, entre 8 et 21 mois, apparaissent des lymphomes le plus souvent immunoblastiques diffus à grandes cellules, se présentant comme des masses abdominales d'extension rapide [3]. Ces lymphomes monoclonaux ont un indice mitotique élevé et, dans $50 \%$ des cas, un réarrangement du gène c-myc. Un tel réarrangement n'est pas observé chez les souris qui développent des lymphomes différenciés oligo-polyclonaux, suggérant que l'expression du transgène est l'agent principal de ces néoplasies de faible malignité alors que des événements ultérieurs impliquant souvent le gène $c-m y c$, dont la synergie avec $b c l-2$ est bien établie [4], seraient nécessaires à l'apparition des lymphomes immunoblastiques de haute malignité. Ainsi sont reproduits, chez ces animaux transgéniques, les stades évolutifs de la progression tumorale des lymphomes humains provoqués par la translocation $\mathrm{t}(14 ; 18)$.

[1. Hockenbery D, et al. Nature 1990 ; 348 : 334-6.]

[2. McDonnell TJ, et al. Cell 1989 ; 57 : 79-88.]

[3. McDonnell TJ, et al. Nature 1991 ; 349 : 254-6.]

[4. Strasser A, et al. Nature 1990 ; 348 : 331-3.] 